\title{
Giant Left Atrial Myxoma: Cause for Position- Dependent Nocturnal Dyspnea and Cardiac Murmur
}

\author{
Julia Hillebrand $^{1}$ Sven Martens ${ }^{1}$ Andreas Hoffmeier ${ }^{1}$
}

\author{
Address for correspondence Dr. Julia Hillebrand, MD, Department of \\ Cardiothoracic Surgery - Division of Cardiac Surgery, University \\ Hospital of the Westfaelische Wilhelms-University Muenster, Albert- \\ Schweitzer-Campus 1, D-48159 Muenster, Germany \\ (e-mail: julia.hillebrand@ukmuenster.de).
}

\begin{abstract}
Keywords

- myxoma

- heart failure

- pathological

Primary tumors of the heart are rare. The majority of them are benign, the left atrial myxoma being the most frequent one. Clinical appearance varies from unapparent to life-threatening complications such as stroke, acute heart failure, or even sudden death. Diagnosis and consecutive surgical treatment strongly depend on the clinical symptoms, but their extent does not correlate with the risk for serious complications. Therefore, patients with variable clinical presentation can be especially endangered. Consequent diagnostic and immediate operative therapy is essential.
\end{abstract}

\section{Introduction}

Primary tumors of the heart are rare. The majority of cardiac tumors are benign, the left atrial myxoma being the most frequent one. Clinical appearance varies from unapparent to life-threatening complications such as stroke, acute heart failure, or even sudden death. ${ }^{1-5}$ Diagnosis and consecutive surgical treatment strongly depend on the clinical symptoms, but their extend does not correlate with the risk for serious complications. ${ }^{1}$ Therefore, patients with variable clinical presentation can be especially endangered. Consequent diagnostic and immediate operative therapy is essential.

\section{Case Description}

We report on surgical treatment of a 39-year-old woman, who had recurrent position-dependent attacks of nocturnal dyspnea. The patient was in a good general condition and her comorbidity was arterial hypertonia and diabetes mellitus. At the time of hospital admission, laboratory findings showed a microcytic anemia; therefore, the patient was treated with folic acid and vitamin B6. Furthermore, the chest X-ray demonstrated signs of pulmonary congestion and pneumonic infiltration. The leukocyte count was within the normal range, whereas C-reactive protein was elevated by $12.6 \mathrm{mg} / \mathrm{dL}$.
Initially, there were no more pathological findings and the presumption diagnosis was a bronchopulmonary infection. However, an inclination toward the left as in auscultation for the mitral valve led to dyspnea. To clarify, a cardiac involvement further diagnosis comprised a cardiac echocardiography. This examination showed a giant left atrial tumor with a length of approximately $8.7 \mathrm{~cm}^{2}$, prolapsing into the left ventricle.

Therefore, urgent cardiac surgery was indicated. After induction of general anesthesia, we performed median sternotomy. Extracorporeal circulation was installed via bicaval connection for venous drainage and cannulation of the ascending aorta for the arterial inflow. The access to the left atrial tumor was performed transseptally. The tumor mass was adherent to the interatrial septum. Therefore, excision of the membranous septum was needed for complete resection. In addition, loose tumorous fragments had to be removed from the bottom of the left atrium as the tumor fell apart during handling, which is not uncommon and one of the principal dangers of this disease. - Fig. 1 shows the extracted tumor $\left(\sim 8.0 \times 4.0 \mathrm{~cm}^{2}\right)$ and one loose fragment. Histopathological examination confirmed the presumed diagnosis of a myxoma. The interatrial septum was reconstructed by the implantation of a bovine pericardial patch. Surgical procedure and postoperative course were without further complication. After 6 days, the patient left hospital in a good clinical condition. received

September 30, 2016

accepted

November 10, 2016
DOI http://dx.doi.org/

10.1055/s-0036-1597987. ISSN 2194-7635. (c) 2017 Georg Thieme Verlag KG Stuttgart · New York

\section{License terms}

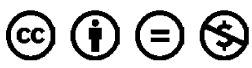




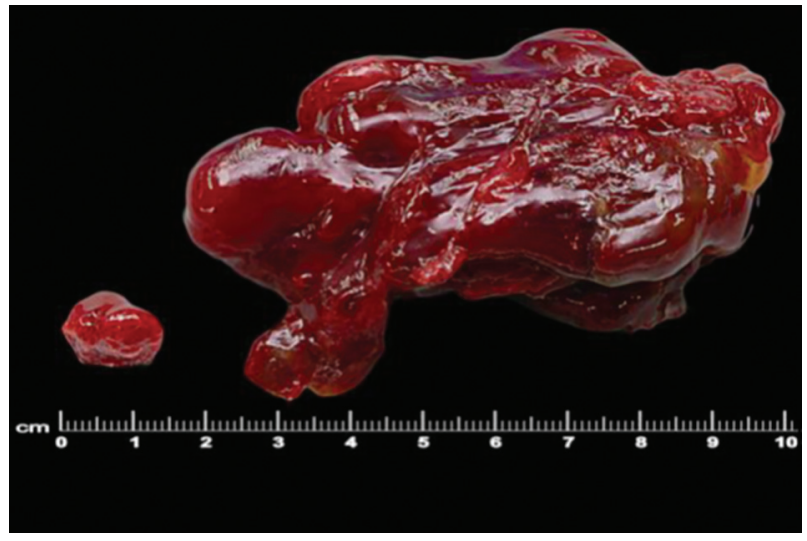

Fig. 1 Giant left atrial myxoma.

\section{Discussion}

A giant left atrial myxoma can be associated with life-threatening complications. ${ }^{1-5}$ However, the extent of clinical impairment does not correlate with the risk for secondary complications. ${ }^{1}$ In 2010, Cabrera et al described that even asymptomatic patients with giant atrial myxoma can be affected by embolic complications or sudden death in up to $15 \%{ }^{2}$ Hence, a consequent diagnostic investigation, differential diagnosis, and prompt surgical treatment are of immense importance.

Among other things, clinical presentation of patients with left atrial myxoma strongly depends on tumor size and localization. Obstruction of the mitral valve, mimicking stenosis often occurs. ${ }^{1,3}$ Nevertheless, diagnosis of left atrial myxoma is still challenging in patients suffering from unspe- cific symptoms or in cases of lacking symptoms of heart failure. Even murmur-free cases have been described. ${ }^{3}$

\section{Conclusion}

The presented case demonstrates another diagnostic aspect in a patient with myxoma, as symptoms were position dependent (inclination toward the left) and nocturnal dyspnea occurred consecutively. Every patient suffering from position-dependent symptoms such as dyspnea or syncopes should be examined by echocardiography, to exclude a causal cardiac tumor. Furthermore, it can be helpful to perform auscultation in different positions. It underlines that accurate anamnesis is essential and that cardiac tumors should be taken into account in the case of varying clinical symptoms such as nocturnal attacks of dyspnea or position-dependent cardiac murmur.

\section{References}

1 Hoffmeier A, Sindermann JR, Scheld HH, Martens S. Cardiac tumors-diagnosis and surgical treatment. Dtsch Arztebl Int 2014;111(12):205-211

2 Cabrera H, Trindade e Silva LP, Gamboa C. Heart failure and syncope due to a giant atrial myxoma. Rev Port Cardiol 2010; 29(2):315-319

3 Nishizaki Y, Yamagami S, Myojin M, et al. A murmur-free giant myxoma discovered incidentally on abdominal ultrasonography. Intern Med 2013;52(22):2529-2531

4 Maintz D, Gunia S, Baumgart P, Hoffmeier A, Fischbach R. Acute myocardial infarction as the first manifestation of left atrial myxoma. AJR Am J Roentgenol 2004;183(6):1838-1839

5 Akhundova A, Samedov F, Cincin A, et al. Giant left atrial myxoma with dual coronary supply presenting with recurrent stroke. Herz $2015 ; 40(2): 318-320$ 